\title{
Primary care emergencies: Improved confidence in clinical and non-clinical members of the multidisciplinary team using a simulation programme
}

\author{
Elizabeth Iris Lamb, ${ }^{1,2}$ Nichola Jenkins, ${ }^{3}$ Philippa Male, ${ }^{1,2,3}$ James McFetrich, ${ }^{3}$ Maria Towart, ${ }^{1}$ Mark Sudlow ${ }^{2,3}$ \\ 1. Northumbria Primary Care \\ 2. Newcastle University \\ 3. Northumbria Healthcare NHS Foundation Trust \\ Correspondence to: Elizabeth.Lamb3@nhs.net \\ Twitter: @DrLilyLamb
}

Word count: 998

\section{Background:}

Emergencies occur infrequently in primary care, and when they do can be daunting for those involved, particularly those who have a non-clinical background. Previous work has described the importance of extending Basic Life Support training to include management of emergencies using the available team and facilities.[1] Simulating waiting room emergencies has been demonstrated to increase confidence in the clinical team in managing these challenging situations,[2] but we are not aware of publications supporting the use of simulation in the wider primary care team including non-clinical colleagues.

Northumbria Primary Care (NPC) is an innovative collaboration between six General Practices and Northumbria Healthcare NHS Foundation Trust (NHFT), serving a population of over 40,000 patients. With the aim of increasing confidence in management of potential encountered medical emergencies in primary care, a shared simulation programme for the clinical and non-clinical multidisciplinary team was developed at the Dinwoodie Assessment and Simulation Hub (DASH) within NHFT. This state-of-the-art facility consists of multiple simulation areas, including consultation rooms and a waiting area, and several low and high-fidelity mannequins, which were used to recreate potential primary care emergencies.

\section{Primary care emergency simulation scenarios:}

A programme was developed based on emergencies that may be encountered in a primary care environment; including meningococcal sepsis in a baby, myocardial infarction leading to cardiac arrest, and anaphylaxis to a vaccine (Table 1).

We developed the environment (e.g. confined consulting spaces and a crowded waiting room, emergency buzzers to summon assistance) to represent a General Practice Surgery. Both mannequins and actors were used in the scenarios to add realistic context and situational challenges. An actor playing a very anxious mother held the simulator baby, and she added to the challenge of the scenario by refusing to wait for the ambulance. The simulator patient with a myocardial infarction was accompanied an angry and disruptive wife, and arrested in a waiting room full of other patients. Available equipment mirrored that available in primary care.

General Practitioners, Nurse Practitioners, Practice Nurses, Practice Managers and Receptionists representing all NPC practices attended. Teams were created including members from each professional role, 
and each team rotated through each simulated scenario. The teams had to work together to manage both the patient's condition and the actors' distress in real time until paramedics arrived.

Stations were facilitated by clinicians with experience of medical education and simulated practice. Each scenario was followed by a team debrief where delegates discussed learning from the scenario. This included clinical management but also human factors around awareness and availability of equipment, communication and team resource management. There was an opportunity to re-run parts of the scenario if the team desired.

\section{Impact of simulation on team confidence:}

Thirty-one staff attended the simulation programme including receptionists $(n=5)$, practice nurses $(n=9)$, managerial staff $(n=5)$, general practitioners $(n=7)$ and nurse practitioners $(n=5)$.

Each attendee completed a questionnaire prior to and following the simulation programme. They were asked to rate their confidence on a 10 point Likert scale of $1-10$, according to four statements: 'I feel confident to manage emergencies as they arise in my practice'; 'I am confident with using medication in an emergency'; 'I understand my role in the team in managing emergencies' and 'I am aware of the protocols to be followed in managing common emergencies'.

Reported confidence in managing emergencies improved significantly in all staff following the simulation programme from a pre-course mean(SE) of $6.33(0.26)$ to a post-course mean of $8.52(0.14)(p<0.0001)$. Significant improvements in understanding role within the team (mean(SE) 7.37(0.39) vs. 9.07(0.18), p<0.001) and in awareness of protocols to be followed in an emergency (mean(SE) 7.07(0.39) vs. 8.54(0.31), $p<0.01$ ) were also reported from pre-course to post-course. A mean(SE) score of $9.8(0.09)$ was given in response to the question 'did you find the session helpful?'

Qualitative data gathered through free-text feedback from participants emphasised the value of interactivity, team-working and realism in support of the learning process. Learners recognised the crucial role that nonclinical staff play in the management of emergencies in the GP setting, and that they were particularly skillful at dealing with the distressed relatives. They identified issues with variation in equipment type and location between practices, which they felt made emergency management more difficult. Clinical staff particularly valued the opportunity to calculate drug doses and draw up medication under simulated emergency pressure.

\section{Discussion}

Simulated waiting room emergencies provided a safe and supportive environment for delegates to work together as a multidisciplinary team to manage infrequently occurring emergency scenarios. The session was well received by staff with reassuring results showing improved confidence in all areas considered. However statistics included are for illustrative purposes only as the sample size is small and there were no controls. Whilst there is limited previously published data on the use of simulation training in primary care, our results support similar recently published data from elsewhere in the UK.[2] Our study builds on this work, by demonstrating, to our knowledge for the first time, benefit from the inclusion of non-clinical practice managers and receptionists as part of primary care simulation programme.

Learning has clearly translated into the clinical environment following this programme. As a direct result, administrative teams in the practices were given further training in communication in challenging situations, changes were made to standardise the availability of emergency medical equipment, protocols were included within emergency boxes, and the location of equipment was highlighted to all staff. Similar translations from simulated to real practice in primary care have recently been reported.[3] 
We conclude that using simulation to train our clinical and non-clinical staff in managing primary care emergencies improves their confidence, and allowed them to identify and improve key areas of team performance. This should translate into improved patient care in a situation where timely and appropriate intervention is critical. We will be running this session for our practices regularly in the future, with additional scenarios including trauma in an elderly patient and acute severe asthma. We recommend that other primary care organisations consider the use of simulation in training their staff to manage emergencies.

\section{References}

1 Eastwick-Field P. No more tick box resuscitation training: simulation in the surgery. British Journal of General Practice 2017;67:25.

2 Forde E, Bromilow J, Wedderburn C. Practical management of emergencies in primary care: taking simulation out of the classroom and into real-life environments. BMJ Simulation and Technology Enhanced Learning 2018;4:43-4.

3 Forde E, Bromilow J, Jackson S, et al. Managing emergencies in primary care: does real-world simulation-based training have any lasting impact? BMJ Simulation and Technology Enhanced Learning 2019;5:57-8.

\section{Funding}

This research received no specific grant from any funding agency in the public, commercial or not-for-profit sectors.

\section{Competing interests}

None of the authors have competing interests to declare.

\section{Author contributions}

All authors were responsible for developing the concept of using simulation to train the wider primary care team, each assisted with designing the curriculum and planning the scenarios, and with running the event on the day. Elizabeth Lamb was responsible for designing the confidence rating tool, for analyzing the data, and for writing the report with assistance from the other authors. 
Table 1: Scenarios used in the waiting room emergencies simulation programme

\begin{tabular}{|c|c|c|}
\hline Scenario & Outcomes & Description \\
\hline $\begin{array}{l}\text { Meningococcal Sepsis } \\
\text { in a baby }\end{array}$ & $\begin{array}{l}\text { Candidates will be aware of the signs } \\
\text { of meningococcal sepsis: pyrexia, lethargy, } \\
\text { tachycardia, non-blanching rash. } \\
\text { Candidates identify the emergency drugs } \\
\text { for managing meningococcal sepsis, } \\
\text { confirm the appropriate doses and treat the } \\
\text { patient appropriately. } \\
\text { Candidates work effectively as a team to } \\
\text { stabilise the patient. }\end{array}$ & $\begin{array}{l}\text { A mother (role player) brings in her 6-month old baby } \\
\text { (mannequin) to see the GP. She is floppy and lethargic. } \\
\text { The candidate assesses the baby in a simulated } \\
\text { consulting room, finds her to be pyrexial, and on removing } \\
\text { her clothing finds a non-blanching rash on her torso. } \\
\text { Candidates should identify the rash and pyrexia as being } \\
\text { signs of meningococcal sepsis and proceed to activate } \\
\text { the 'red button' to obtain assistance, call an ambulance } \\
\text { and administer intramuscular benzylpenicillin. They also } \\
\text { need to manage the mother's anxiety about waiting for an } \\
\text { ambulance and repeated attempts to take the baby and } \\
\text { leave the practice. The paramedics arrive after } 10 \\
\text { minutes. }\end{array}$ \\
\hline $\begin{array}{l}\text { Chest pain leading to } \\
\text { cardiac arrest in an } \\
\text { adult male }\end{array}$ & $\begin{array}{l}\text { - Candidates will be aware of the presenting } \\
\text { symptoms of a Myocardial Infarction (MI). } \\
\text { The reception team recognise that they } \\
\text { should call } 999 \text { if they are concerned a } \\
\text { patient is having an MI. } \\
\text { Candidates will be able to recall the Basic } \\
\text { life support (BLS) protocol and put it into } \\
\text { practice in a simulated environment. } \\
\text { Candidates will be confident in using the } \\
\text { automatic external defibrillator (AED). }\end{array}$ & $\begin{array}{l}\text { A } 60 \text { year old male (role player) walks into the simulated } \\
\text { waiting room with his wife complaining of severe chest } \\
\text { pain, clutching his chest and looking clammy. He asks the } \\
\text { receptionist if he can be seen by a clinician and then } \\
\text { proceeds to collapse. A mannequin then takes his place. } \\
\text { The receptionist should activate the 'red button' to call for } \\
\text { help, dial } 999 \text { and obtain the emergency equipment and } \\
\text { automated defibrillator. The assisting team should identify } \\
\text { that the patient is in cardiac arrest and initiate the BLS } \\
\text { protocol, using the AED and following the instructions it } \\
\text { gives until paramedics arrive } 8 \text { minutes later. The wife } \\
\text { (role player) is very distressed throughout the scenario } \\
\text { and members of the team should be allocated to } \\
\text { supporting her during the resuscitation attempt. }\end{array}$ \\
\hline $\begin{array}{l}\text { Anaphlaxis to a flu } \\
\text { vaccine in an adult } \\
\text { female }\end{array}$ & $\begin{array}{l}\text { Candidates will be aware of the signs of } \\
\text { anaphylaxis: urticarial rash, oedema, } \\
\text { respiratory difficulty, collapse. } \\
\text { Candidates identify the emergency drugs } \\
\text { for managing this, confirm the appropriate } \\
\text { doses and treat the patient appropriately. } \\
\text { Candidates work effectively as a team to } \\
\text { stabilise the patient. }\end{array}$ & $\begin{array}{l}\text { A } 47 \text { year old female (high-fidelity mannequin) is visiting } \\
\text { the practice nurse for a flu vaccine due to a recent } \\
\text { diagnosis of COPD. She is known to have a nut allergy. A } \\
\text { candidate administers the vaccine and within a minute the } \\
\text { patient becomes unwell with breathing difficulty, lip and } \\
\text { tongue swelling, itch and an urticarial rash. The candidate } \\
\text { should activate the red button to call the team and obtain } \\
\text { the emergency equipment. The team should recognize } \\
\text { that the patient is suffering from anaphylaxis, call } 999 \text { and } \\
\text { proceed to administer intramuscular adrenaline, } \\
\text { hydrocortisone and chlorpheniramine whilst awaiting the } \\
\text { arrival of the paramedics approximately } 8 \text { minutes later. }\end{array}$ \\
\hline
\end{tabular}

